\title{
Quantum dot device tunable from single to triple dot system
}

\author{
M. C. Rogge*, K. Pierz ${ }^{\dagger}$ and R. J. Haug* \\ *Institut für Festkörperphysik, Leibniz Universität Hannover, Appelstrasse 2, 30167 Hannover, Germany \\ $\dagger$ Physikalisch-Technische Bundesanstalt, Bundesallee 100, 38116 Braunschweig, Germany
}

\begin{abstract}
We present a lateral quantum dot device which has a tunable number of quantum dots. Depending on easily tunable gate voltages, one, two or three quantum dots are found. They are investigated in transport and charge detection.
\end{abstract}

Keywords: Quantum dots, Coulomb blockade, single-electron tunneling PACS: 73.21.La, 73.23.Hk, 73.63.Kv

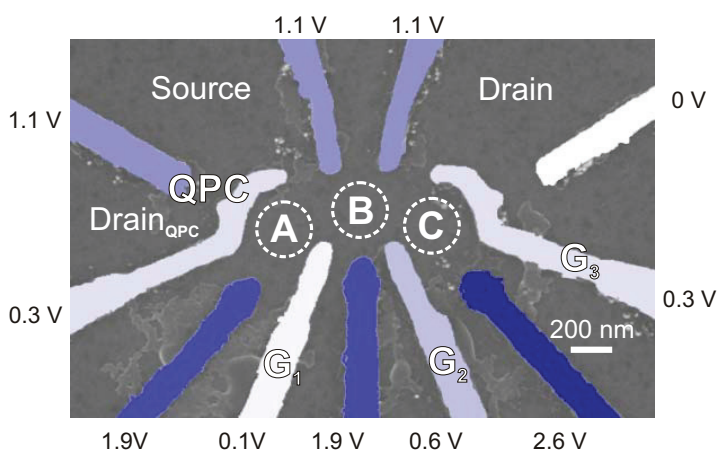

FIGURE 1. SEM-image of the device. Three quantum dots A, B, C can be placed between contacts Source and Drain. A QPC is added for charge detection with a separate Drain $_{Q P C}$ contact. Gates $G_{1}, G_{2}, G_{3}$ were varied during measurements. The color of the gates reflects the bias used for bias cool down.

In recent years, many interesting results have been gained with research on lateral single $[1,2,3]$, double $[4,3]$ and triple dots (eg. [5, 6, 7, 8, 9]). In most cases, the devices fabricated for those investigations were specifically designed for a fixed number of quantum dots. We want to show, that a single device can be used instead, reducing the effort of sample production and measurement setup. We present a quantum dot device which has a tunable number of quantum dots depending on gate voltages. A maximum number of three quantum dots is possible but can be easily reduced to two or one. Therefore the need to built many different devices with different numbers of quantum dots is overcome with just one tunable device.

We defined the device laterally on a GaAs/AlGaAs heterostructure with a two dimensional electron gas (2DEG) $85 \mathrm{~nm}$ below the surface. The electron mobility at liquid helium temperature is $\mu=95 \mathrm{~m}^{2} / \mathrm{Vs}$. The electron density is $n=2.9 * 10^{15} \mathrm{~m}^{-2}$. Several $\mathrm{Cr} / \mathrm{Au}$ top gates are fabricated on the surface in order to de-

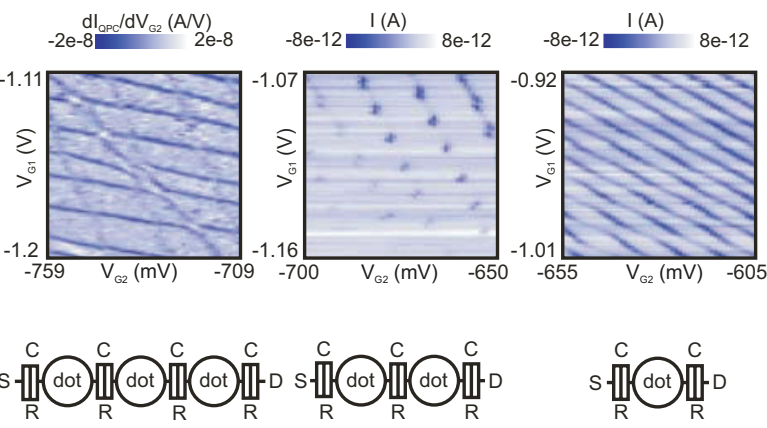

FIGURE 2. Left: charge detection for quite negative voltages at $G_{1}$ and $G_{2}$. Three sets of lines are visible as they are expected for a triple quantum dot. Center: current through the device for slightly higher voltages. A regular pattern of spots indicates a serial double dot. Right: With even more positive voltages the pattern changes to parallel solid lines as expected for a single quantum dot. The schemes below the measurements show the configuration of the system with both the triple dot and the double dot connected in series to Source and Drain. The dots are coupled via capacitances $C$ and resistances $R$.

fine the quantum dots. In Fig. 1 an SEM-image of the sample is presented. The top gates are arranged such that three quantum dots $\mathrm{A}, \mathrm{B}$ and $\mathrm{C}$ can be placed between a Source and a Drain contact. In addition a quantum point contact (QPC) is located next to the dots for charge de-

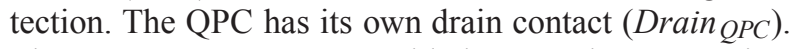
The measurements presented below are done sweeping gates $G_{1}, G_{2}$ and $G_{3}$. For noise reduction, we used a bias cool down [10]. The voltages were applied individually for each gate ranging from $0 \mathrm{~V}$ to $2.6 \mathrm{~V}$ as indicated by the color of the gates.

With this device we can change the number of quantum dots. For this purpose one has to change voltages at only two gates. Those are the central gates $G_{1}$ and $G_{2}$. Figure 2 shows three measurements that confirm this tunability. On the left of Fig. 2 the charge detection (the derivative of the QPC current with respect to $V_{G 2}$ ) is plot- 


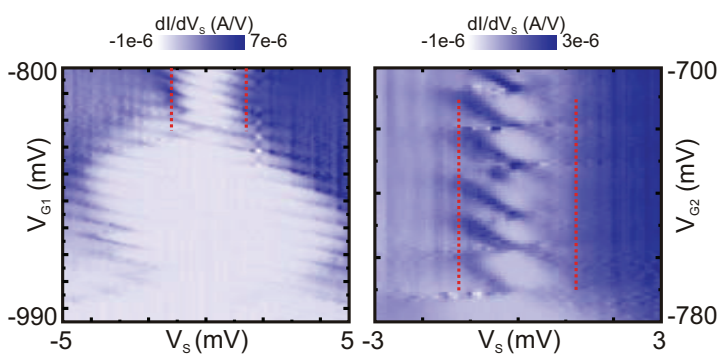

FIGURE 3. Coulomb diamonds as a function of $V_{S}$ and $V_{G 1}$ (left) and $V_{G 2}$ (right) respectively. The left measurement shows diamonds from dot A with a width of approx. $1.4 \mathrm{mV}$ (dotted lines), the right image shows those of $\operatorname{dot} C$ with a similar width of approx. $1.4 \mathrm{mV}$ (dotted lines). In addition, the left image shows one large diamond for dot B (width approx. $5 \mathrm{mV}$ ), which fills out the whole center of the measurement.

ted for relatively negative voltages at gates $V_{G 1}$ and $V_{G 2}$. Here a triple dot is observed as schematically shown below the measurement. As the dots are connected in series, transport is expected only at so called quadruple points $[11,12]$, but with charge detection all the charging lines of the three different dots become visible. And indeed, one can see three sets of lines with different slopes. Those with a small gradient denote charging of quantum $\operatorname{dot} \mathrm{A}$, those with a large gradient belong to quantum dot C. The intermediate slopes appear due to charging of dot B. At the intersections of these lines, anticrossings occur due to finite coupling between the dots. The line for dot $B$ represents the first electron entering the dot. The number of electrons for the other dots is higher as explained below.

At slightly higher voltages the situation is changed. Now the current through the system shows a pattern of spots, which is typical for a serial double dot (see schematic below). In double dots, those spots appear at both sides of an anticrossing, when three charge configurations are degenerate. Therefore they are called triple points. So, just by increasing the voltage at gate $G_{2}$, the number of quantum dots was reduced from three to two, probably by merging the original dots $\mathrm{B}$ and $\mathrm{C}$.

A further increase of $V_{G 1}$ leads to the measurement presented on the right of Fig. 2. Now the pattern observed in transport has changed dramatically. The isolated spots representing triple points have vanished in favor of solid parallel lines. These kind of lines are well known for single quantum dots. They are called Coulomb blockade peaks. In between those lines, transport is Coulomb blocked. This is a nice indication that now all three dots have merged and a large single dot is formed as schematically shown below the measurement.

As mentioned above, the number of electrons for the triple dot case is known for dot B to be one. This is because the charging line for dot $\mathrm{B}$ is the last one visi- ble. No further line appears for more negative voltages. Therefore the dot is completely emptied and the line shows charging with the first electron. The number of electrons for dots $\mathrm{A}$ and $\mathrm{C}$ can only be estimated indirectly from nonlinear measurements. Figure 3 shows two measurements of the current derived by Source voltage $V_{S}$ as a function of $V_{S}$ and $V_{G 1}$ ( $V_{G 2}$ respectively). In the upper part of the left measurement, several Coulomb diamonds are visible. They stem from quantum dot A. The width of those diamonds is roughly $\pm 1.4 \mathrm{mV}$ (dotted vertical lines). This directly transfers via $1.4 \mathrm{meV}=e^{2} / C$ (with $\mathrm{C}$ the total capacitance) to $C \approx 114 \mathrm{aF}$. Assuming a circular disk for the dot, the radius is estimated to $r=C / 8 \varepsilon_{0} \varepsilon_{r} \approx 130 \mathrm{~nm}$. With the electron density $n$ (see above) now the electron number can be estimated to be approx. 150. The same method is used for quantum dot $\mathrm{C}$, whose Coulomb diamonds can be seen on the right of Fig. 3. The Coulomb diamonds are of similar size (dotted lines) and thus a similar number of 150 electrons is found for dot C. Those values are upper limits. Due to the surrounding gates the capacitance of the dots is enlarged compared to a disk in free space. Therefore the actual number of electrons is significantly smaller. We estimate several ten to 100 electrons per dot. This effect is also observed for dot $\mathrm{B}$. The large central diamond on the left of Fig. 3 stems from dot B. With a width of approx. $\pm 5 \mathrm{mV}$ the calculated number of electrons is 12 , twelve times larger than the actual one.

In conclusion we have fabricated a quantum dot device with a tunable number of quantum dots. Depending on gate voltages, one, two or three dots are found. The number of dots as well as their serial arrangement is confirmed with current measurements and charge detection.

\section{REFERENCES}

1. L. P. Kouwenhoven et al., in Mesoscopic electron transport, vol. 345 of Series E, pp. 105-214 (1991).

2. U. Meirav and E. B. Foxman, Semicond. Sci. Technol.11, 255 (1996).

3. R. Hanson et al., Rev. Mod. Phys. 97, 1217 (2007).

4. W. G. van der Wiel et al., Rev. Mod. Phys. 75, 1 (2003).

5. A. Vidan et al., Appl. Phys. Lett. 85, 3602 (2004).

6. L. Gaudreau et al., Phys. Rev. Lett. 97, 036807 (2006).

7. D. Schröer et al., Phys. Rev. B 76, 075306 (2007).

8. M. C. Rogge and R. J. Haug, Phys. Rev. B 77, 193306 (2008).

9. D. Amaha et al., Physica E 40, 1322 (2008).

10. M. Pioro-Ladrière et al., Phys. Rev. B 72, 115331 (2005).

11. M. C. Rogge and R. J. Haug, New Journal of Physics 11, 113037 (2009).

12. G. Granger et al., Phys. Rev. B 82, 075304 (2010). 
AIP Conference Proceedings is copyrighted by AIP Publishing LLC (AIP). Reuse of AIP content is subject to the terms at: http://scitation.aip.org/termsconditions. For more information, see http://publishing.aip.org/authors/rights-and-permissions. 